\title{
Factores asociados al desarrollo de anomalías congénitas en la población neonatal atendida en un hospital de alta complejidad en Colombia: estudio de casos y controles
}

\section{Associated factors with the development of congenital anomalies in the neonatal population attended in a highly complex hospital in Colombia: case-control study}

\section{Daniela Torres-Hernández', Tatiana Fletcher-Toledo', Roberth A. Ortiz-Martínez², $M^{a}$ Amparo Acosta-Aragón ${ }^{3,4 *}$, Luis L. Moreno-Montenegro ${ }^{1}$ y Mayra F. Otalora-Perdomo ${ }^{5}$}

${ }^{1}$ Servicio de Pediatría, Universidad del Cauca, Popayán; ${ }^{2}$ Servicio de Ginecología y Obstetricia, Universidad del Cauca, Popayán; ${ }^{3}$ Servicio de Pediatría, Facultad de Ciencias de la Salud, Universidad del Cauca, Popayán; ${ }^{4}$ Servicio de Pediatría, Hospital Universitario San José, Popayán; ${ }^{5}$ Servicio de Ginecología y Obstetricia, Universidad Libre, Cali. Colombia

\section{Resumen}

Introducción: Los defectos congénitos son un grupo de alteraciones con gran heterogeneidad clínica y etiológica. Su prevalencia en los países en vías de desarrollo alcanza aproximadamente un 7\%. En Colombia, un 17\% de los niños menores de 1 año mueren a causa de anomalías congénitas. Objetivo: Determinar los factores de riesgo asociados a anomalías congénitas en neonatos del Departamento del Cauca atendidos en un hospital de alta complejidad. Método: Estudio de casos y controles en el Hospital Universitario San José, de Popayán, Colombia. Se incluyeron 174 recién nacidos, con una distribución 1:1 para 87 casos y 87 controles, entre julio de 2018 y julio de 2019. Las variables de interés fueron registradas en un instrumento semiestructurado diseñado por los investigadores. Los datos obtenidos fueron analizados usando métodos de estadística descriptiva, prueba exacta de Fisher y modelos de regresión logística binomial (odds ratio [OR] con intervalo de confianza del 95\% [IC95\%]). Resultados: Las anomalías congénitas más frecuentes fueron del sistema cardiovascular (40,23\%), renales (24,14\%) y del sistema nervioso central $(13,79 \%)$. Las variables que se asociaron a anomalías congénitas fueron la educación de la madre (OR: 2,40; IC95\%: 1,23-4,68), la educación del padre (OR: 2,93; IC95\%: 1,445,93), el antecedente de cesárea (OR: 3,3; IC95\%: 1,76-6,42), la hemorragia en el primer trimestre (OR: 6,15; IC95\%: 1,3228,63) y el antecedente de malformación en un embarazo previo (OR: 4,05; IC95\%: 1,08-15,07). Conclusiones: De acuerdo con los resultados del presente estudio, para tratar de reducir la incidencia de las anomalías congénitas se deben intervenir los factores de riesgo modificables, como la educación de los padres, tratar oportunamente las patologías maternas asociadas al sangrado del primer trimestre y realizar consejería genética a los padres con antecedente de anomalías congénitas en embarazos previos. Lo anterior podría lograrse al mejorar la calidad y la adherencia al control prenatal.

Palabras clave: Anomalías congénitas. Factores genéticos. Factores ambientales.

Correspondencia:

*Mำ Amparo Acosta-Aragón E-mail: morin1924@gmail.com; maragon@unicauca.edu.co

0048-766X / @ 2021 Sociedad Chilena de Obstetricia y Ginecología. Publicado por Permanyer. Éste es un artículo open access bajo la licencia CC BY-NC-ND (https://creativecommons.org/licenses/by-nc-nd/4.0/).
Disponible en internet: 02-08-2021 Rev Chil Obstet Ginecol. 2021;86(3):301-308 www. rechog.com 


\section{Abstract}

Introduction: Congenital defects are a group of alterations with great clinical and etiological heterogeneity. The prevalence in developing countries is approximately $7 \%$. In Colombia, $17 \%$ of children under 1 year of age die from congenital anomalies. Objective: To determine the risk factors associated with congenital anomalies in neonates from the Department of Cauca treated in a highly complex hospital. Method: Case-control study at the San José de Popayan University Hospital, Colombia. 174 newborns entered the study, with a 1: 1 distribution for 87 cases and 87 controls, between July 2018 and July 2019. The variables of interest were recorded in a semi-structured instrument designed by the researchers. The data obtained were analyzed using descriptive statistical methods, Fisher's exact test and binomial logistic regression models (OR with $95 \% \mathrm{Cl}$ ). Results: The most frequent congenital anomalies were those of the cardiovascular system $(40.23 \%)$, renal $(24.14 \%)$ and central nervous system (13.79\%). The variables associated with congenital anomalies were mother's education (OR 2.40; 95\% Cl: 1.23-4.68), father's education (OR 2.93 95\% Cl: 1.44-5.93), history of cesarean section (OR $3.3 \mathrm{Cl}$ 95\%: 1.76-6.42), first trimester bleeding (OR 6.15 95\% Cl: 1.32-28.63); history of malformation (OR: 4,05; 95\% Cl: 1.08-15.07). Conclusions: Based on the results of the present study and to try to reduce the incidence of congenital anomalies, modifiable risk factors should be intervened, such as parental education, timely treatment of maternal pathologies associated with 1-trimester bleeding and perform genetic counseling to parents with a history of congenital anomalies in previous pregnancies. This could be achieved by improving quality and adherence to prenatal care.

Key words: Congenital anomalies. Genetic factors. Environmental factors.

\section{Introducción}

Las anomalías congénitas contribuyen de manera significativa a la morbilidad y la mortalidad infantiles. En América Latina representan la segunda causa de muerte en los menores de 5 años, al igual que en Colombia, donde son la segunda causa de muerte en los menores de 1 año, provocando el $20,8 \%$ de las muertes ${ }^{\dagger}$. En todo el mundo se estima que al menos 7,9 millones de niños nacen cada año con anomalías congénitas graves, las cuales causan 3,2 millones de discapacidades al año. Sin embargo, la gran diversidad de anomalías existentes, y en algunos casos la imposibilidad de establecer el diagnóstico prenatal, dificulta la obtención de datos precisos sobre la prevalencia de este fenómeno? ${ }^{2}$.

Los defectos congénitos forman un grupo heterogéneo de trastornos que pueden obedecer a la presencia de ciertos factores: ambientales (teratógenos), genéticos de origen monogénico o poligénico, anomalías cromosómicas numéricas o estructurales, o una combinación de factores genéticos y ambientales (herencia multifactorial) ${ }^{3}$.

Dentro de los factores de riesgo asociados a las anomalías congénitas se reportan como los más frecuentes la edad materna avanzada, la edad paterna igual o mayor de 40 años, el no consumo preconcepcional de ácido fólico, el consumo de alcohol, algunos medicamentos (captopril, ácido valproico, litio, ácido retinoico, tetraciclinas), las radiaciones, los químicos teratogénicos, la consanguinidad y la endogamia ${ }^{4-8}$.
Hacen falta más estudios que permitan identificar los factores que intervienen en el desarrollo de estas patologías, lo que permitiría diseñar estrategias de prevención y dar un manejo oportuno y adecuado.

El presente estudio tiene como objetivo determinar los factores de riesgo asociados a la ocurrencia de anomalías congénitas en recién nacidos en un hospital de alta complejidad del departamento del Cauca, Colombia.

\section{Método}

Se realizó un estudio observacional de casos y controles para determinar los factores asociados a la ocurrencia de anomalías congénitas. El estudio se llevó a cabo entre julio de 2018 y julio de 2019 en el Hospital Universitario San José, centro de referencia en el departamento del Cauca, en el suroccidente de Colombia. Se incluyeron en el estudio 174 recién nacidos. Se definió como caso al neonato (vivo o muerto) con peso $\geq 500 \mathrm{~g}$ con una o más anomalías congénitas. Cabe aclarar que estas se dividen en dos categorías: 1) mayores, definidas como defectos que, si no son corregidos, comprometen significativamente el funcionamiento corporal o reducen la expectativa normal de vida; y 2) menores, definidas como defectos que no comprometen seriamente la forma ni la funcionalidad corporal ${ }^{9}$. Los controles se definieron como neonatos vivos a término sin anomalías que nacieron alrededor de las 48 horas del caso. 
Se excluyeron los neonatos cuyos padres no aceptaron entrar en la investigación y los casos detectados después de 48 horas del nacimiento.

El diagnóstico de anomalía congénita fue realizado inicialmente por el pediatra o el residente de pediatría a cargo del caso y posteriormente por las investigadoras y la genetista clínica, confirmado con ayudas diagnósticas cuando fueron necesarias (imagenología y pruebas genéticas). De acuerdo con los parámetros establecidos, para el tamaño de muestra se tuvo en cuenta un alfa de 0,05 y un beta de 0,20 ; tasa de exposición a consanguinidad del $15 \%$, un OR 2.8 correspondiente al incremento minino del OR de interés según estudios previos ${ }^{10,11}$. Se tomó una relación 1:1 entre casos y controles, con lo que se obtuvo un tamaño de muestra de 87 casos y 87 controles. Se consideró la variable de consanguinidad para el cálculo del tamaño de la muestra, dado que no se tenía información de la frecuencia de las anomalías congénitas en la población de interés y que su relación con las anomalías congénitas ha sido reportada por otros estudios ${ }^{5,6}$.

La edad gestacional al nacimiento se obtuvo de la ecografía del primer trimestre o del examen clínico realizado por el pediatra.

Para el registro de las anomalías se aplicó el formato estándar establecido por el Estudio Colaborativo Latinoamericano de Malformaciones Congénitas (ECLAMC) ${ }^{7}$. Se midieron también variables sociodemográficas, con el fin de obtener información adicional de ambos progenitores: edad, nivel educativo, estrato socioeconómico, ingresos del hogar, zona de residencia, grupo étnico, ocupación, consanguinidad parental, años de convivencia, dificultad para la concepción y exposición a teratógenos durante la concepción (minería, baterías, pesticidas, plomo, mercurio, consumo de alcohol o cigarrillos, tiempo de consumo de cigarrillos, exposición pasiva, otras sustancias psicoactivas, etc.). Como variables biológicas del recién nacido se consideraron el peso, el sexo, la edad de diagnóstico, la condición al alta del servicio, la forma de evidencia diagnóstica y la hemoclasificación.

De igual manera, se midieron variables biológicas relacionadas con el embarazo: edad gestacional al nacimiento, fórmula obstétrica, número de controles prenatales, edad gestacional de ingreso en el control prenatal, peso preconcepcional, ganancia de peso durante el embarazo, diabetes gestacional, hipertensión previa o inducida por el embarazo, hipotiroidismo o hipertiroidismo gestacional o preconcepcional, síndrome convulsivo, toxoplasmosis, rubeola, citomegalovirus, herpes simple, sífilis, virus de la inmunodeficiencia humana $(\mathrm{VIH})$, madre o padre con virus Zika, antecedente de anomalías congénitas, hemorragia en el primer trimestre, medicamentos (ácido fólico, hierro, calcio, multivitaminas combinadas, oligoelementos), vacunación (influenza, tétanos y pertusis), presentación del feto (cefálica o pélvica), tipo de parto, embarazo múltiple, tipo de sangre de los padres, anomalías congénitas en la familia e hijos en común con el padre.

Las variables fueron registradas en un instrumento semiestructurado diseñado por los investigadores ${ }^{6,8,9}$, aprobado por expertos (profesores del Departamento de Pediatría) y ajustado por prueba piloto realizada en mayo de 2018 con 30 pacientes. El análisis de la información se llevó a cabo con el programa Stata versión 15.0.

\section{Análisis estadístico}

Para el análisis estadístico se usaron métodos de estadística descriptiva (frecuencias, porcentaje, promedio y desviación estándar). Las variables se analizaron individualmente desde el punto de vista exploratorio para comprobar la normalidad de su distribución e identificar valores extremos y perdidos que pudieran afectar el resultado. Se utilizó la prueba t de Student para las variables continuas con distribución normal, previo análisis de la varianza. Para las de distribución no normal se utilizaron la prueba $U$ de Mann-Whitney, previa aplicación del test de normalidad de Shapiro-Wilk, y la prueba de ji al cuadrado o el test exacto de Fisher según correspondiera. Para la evaluación de los posibles factores asociados a las anomalías congénitas se realizaron modelos bivariados de regresión logística, determinando la OR y el intervalo de confianza del 95\% (IC95\%).

\section{Consideraciones éticas}

El estudio estuvo regido por las normas bioéticas internacionales vigentes, como el Código de Núremberg, la Declaración de Helsinki y el Reporte Belmont. Además, se restringe a las normas del Código Civil Colombiano y la Resolución 8430 de 1993 del Ministerio de Salud. El protocolo de investigación fue aprobado por el Comité de Ética del Hospital Universitario San José de Popayán y de la Universidad del Cauca. Todos los participantes firmaron un consentimiento informado previo al inicio del estudio.

\section{Resultados}

Se analizaron en total 87 casos y 87 controles. Las variables sociodemográficas de ambos grupos se describen en la tabla 1. 
Rev Chil Obstet Ginecol. 2021;86(3)

Tabla 1. Distribución de frecuencias según las variables sociodemográficas de los casos y controles

\begin{tabular}{|c|c|c|c|}
\hline Variables & $\begin{array}{l}\text { Anomalías congénitas } \\
\text { (87 casos) } \\
\text { n (\%) }\end{array}$ & $\begin{array}{c}\text { Sin anomalías congénitas } \\
\text { (87 controles) } \\
n(\%)\end{array}$ & $\mathrm{p}$ \\
\hline $\begin{array}{l}\text { Educación de la madre } \\
6 \text { años o más } \\
5 \text { años o menos }\end{array}$ & $\begin{array}{l}52(59,77) \\
35(40,23)\end{array}$ & $\begin{array}{l}68(78,16) \\
19(21,84)\end{array}$ & 0,009 \\
\hline $\begin{array}{l}\text { Educación del padre } \\
6 \text { años o más } \\
5 \text { años o menos }\end{array}$ & $\begin{array}{l}54(62,07) \\
33(37,93)\end{array}$ & $\begin{array}{l}72(82,76) \\
15(16,24)\end{array}$ & 0,002 \\
\hline $\begin{array}{l}\text { Raza de la madre } \\
\text { Blanca/mestiza } \\
\text { Indígena } \\
\text { Negra }\end{array}$ & $\begin{array}{c}66(75,86) \\
20(22,99) \\
1(1,15)\end{array}$ & $\begin{array}{c}71(81,61) \\
12(13,79) \\
4(4,60)\end{array}$ & $0,16^{*}$ \\
\hline $\begin{array}{l}\text { Raza del padre } \\
\text { Blanca/mestiza } \\
\text { Indígena } \\
\text { Negra }\end{array}$ & $\begin{array}{l}71(81,61) \\
15(17,24) \\
1(1,15)\end{array}$ & $\begin{array}{l}74(85,06) \\
8(9,20) \\
5(5,75)\end{array}$ & $0,09 *$ \\
\hline $\begin{array}{l}\text { Consanguinidad } \\
\text { No } \\
\text { Sí }\end{array}$ & $\begin{array}{c}81(93,10) \\
6(6,90)\end{array}$ & $\begin{array}{c}86(98,85) \\
1(1,15)\end{array}$ & $0,11^{*}$ \\
\hline $\begin{array}{l}\text { Antecedente de cesárea } \\
\text { Sin antecedente } \\
\text { Con antecedente }\end{array}$ & $\begin{array}{l}42(48,28) \\
45(51,72)\end{array}$ & $\begin{array}{l}66(75,86) \\
21(24,14)\end{array}$ & 0,00 \\
\hline $\begin{array}{l}\text { Antecedente de abortos } \\
\text { Uno o ninguno } \\
\text { Dos o más }\end{array}$ & $\begin{array}{c}82(42,25) \\
5(5,75)\end{array}$ & $\begin{array}{c}85(97,70) \\
2(2,30)\end{array}$ & $0,44^{*}$ \\
\hline $\begin{array}{l}\text { Enfermedad crónica } \\
\text { No } \\
\text { Sí }\end{array}$ & $\begin{array}{l}69(79,31) \\
18(20,69)\end{array}$ & $\begin{array}{l}70(80,46) \\
17(19,54)\end{array}$ & 0,85 \\
\hline $\begin{array}{l}\text { Hemorragia en el primer trimestre } \\
\text { No } \\
\text { Sí }\end{array}$ & $\begin{array}{l}76(87,36) \\
11(12,64)\end{array}$ & $\begin{array}{c}85(97,70) \\
2(2,30)\end{array}$ & $0,00^{*}$ \\
\hline $\begin{array}{l}\text { Fuma } \\
\text { No } \\
\text { Sí }\end{array}$ & $\begin{array}{c}86(98,85) \\
1(1,15)\end{array}$ & $\begin{array}{c}85(97,70) \\
2(2,30)\end{array}$ & $0,56^{*}$ \\
\hline Sexo del neonato & & & \\
\hline Masculino & $46(52,87)$ & $41(47,13)$ & 1,00 \\
\hline Femenino & $41(47,13)$ & $46(52,87)$ & \\
\hline $\begin{array}{l}\text { Controles prenatales } \\
\text { Cuatro o más } \\
\text { Tres o menos }\end{array}$ & $\begin{array}{l}70(80,46) \\
17(19,54)\end{array}$ & $\begin{array}{l}72(82,76) \\
15(17,24)\end{array}$ & 0,69 \\
\hline $\begin{array}{l}\text { Antecedente de malformaciones } \\
\text { No } \\
\text { Sí }\end{array}$ & $\begin{array}{l}76(87,36) \\
11(12,64)\end{array}$ & $\begin{array}{c}84(96,55) \\
3(3,45)\end{array}$ & $0,04^{*}$ \\
\hline $\begin{array}{l}\text { Endogamia } \\
\text { No } \\
\text { Sí }\end{array}$ & $\begin{array}{l}56(64,37) \\
31(35,63)\end{array}$ & $\begin{array}{l}55(63,22) \\
32(36,78)\end{array}$ & 0,87 \\
\hline
\end{tabular}

Para la diferencia entre los grupos se usaron las pruebas exacta de Fisher $\left({ }^{*}\right)$ y ji al cuadrado.

El promedio de edad de las madres fue de 26,67 \pm 7,00 años, mientras que el de los padres fue de 31,34 $\pm 7,90$ años. La mayor parte de la población era de área rural, con un $52,87 \%$. El $40,23 \%$ de las madres tenían un nivel educativo de menos de 5 años de escolaridad, mientras que en los padres el porcentaje era 
del $37,93 \%$. La raza más frecuente del padre y de la madre fue blanco/mestizo, en un $81,61 \%$ y un $75,86 \%$ de los casos, respectivamente. Había consanguinidad en el 6,90\%, y la percepción propia sobre la consanguinidad como algo anormal fue del $93,10 \%$. En contraste, la endogamia se encontró en el $35,63 \%$ de la población.

La paridad de las mujeres fue de tres o menos en el $87,36 \%$, y los antecedentes de cesárea fueron del $51,72 \%$. De las mujeres encuestadas, el 5,75\% habían presentado dos o más abortos y el $33,33 \%$ tenían antecedentes de mortinatos. Se encontró un caso de VIH y otro de sífilis.

El $4,60 \%$ de las mujeres presentaron dificultades para la concepción. El $80,46 \%$ realizaron tres o más controles prenatales. El 12,64\% refirieron antecedentes de anomalías congénitas en embarazos previos. El $12,64 \%$ presentaron hemorragia en el primer trimestre. El 20,69\% tenían antecedentes de enfermedad crónica. Menos del $2 \%$ de las mujeres manifestaron haber fumado y consumido alcohol durante la gestación, y el $4,60 \%$ se consideraban fumadoras pasivas. Como última observación, ninguna consumió sustancias psicoactivas (Tabla 1).

El $44,83 \%$ de las gestantes tuvieron el parto por cesárea. El promedio de peso de los recién nacidos fue de $2996,87 \pm 644,63 \mathrm{~g}$. En cuanto al sexo, el $52,87 \%$ fueron niños y el $47,13 \%$ fueron niñas. Con respecto al tamaño al nacer, el $76,44 \%$ tenían un peso adecuado al nacimiento y el $83,33 \%$ nacieron de término. Finalmente, de los pacientes analizados, $3(1,7 \%)$ fallecieron.

Las anomalías congénitas más frecuentes fueron los defectos cardiovasculares, con un $40,23 \%$, seguidos de los defectos del sistema urogenital con un $24,14 \%$ y del sistema nervioso con un $13,79 \%$ (Fig. 1). En la tabla 2 se especifican los defectos congénitos.

Al realizar el análisis de regresión logística se encontró que la consanguinidad y la endogamia no mostraron asociación significativa con las anomalías congénitas (OR: 6,37, IC95\%: 0,75-54,07; y OR: 0,95, IC95\%: 0,51-1,76, respectivamente). Las variables que estuvieron asociadas con la presencia de anomalías congénitas fueron la educación de la madre (OR: 2,40; IC95\%: 1,23-4,68), la educación del padre (OR: 2,93; IC95\%: 1,44-5,93), el antecedente de cesárea (OR: 3,3; IC95\%: 1,76-6,42), la hemorragia del primer trimestre (OR: 6,15; IC95\%: 1,32-28,63) y el antecedente de anomalías congénitas (OR: 4,05; IC95\%: 1,08-15,07) (Tabla 3).
Tabla 2. Tipo de anomalía congénita por sistema

\begin{tabular}{|c|c|c|c|}
\hline Sistema & Total & n & $\%$ \\
\hline Defectos cardiovasculares & 35 & & 40,23 \\
\hline Comunicación interauricular & & 23 & 65,71 \\
\hline Comunicación interventricular & & 12 & 34,29 \\
\hline Defectos urogenitales & 21 & & 24,14 \\
\hline Pielectasia renal & & 13 & 61,90 \\
\hline Hidronefrosis congénita & & 6 & 28,57 \\
\hline Displasia multiquística & & 1 & 4,76 \\
\hline Riñón poliquístico & & 1 & 4,76 \\
\hline Defectos del sistema nervioso & 12 & & 13,79 \\
\hline Espina bífida & & 4 & 33,33 \\
\hline Hoyuelo sacro & & 3 & 25 \\
\hline Ventriculomegalia cerebral & & 3 & 25 \\
\hline Holoprosencefalia & & 1 & 8,33 \\
\hline Hidranencefalia & & 1 & 8,33 \\
\hline $\begin{array}{l}\text { Defectos craneales, faciales } 0 \\
\text { cervicales }\end{array}$ & 6 & & 6,90 \\
\hline Queilopalatosquisis & & 6 & 100 \\
\hline Defectos gastrointestinales & 4 & & 4,60 \\
\hline Atresia duodenal & & 2 & 50 \\
\hline Estenosis intestinal & & 1 & 25 \\
\hline Atresia de esófago & & 1 & 25 \\
\hline $\begin{array}{l}\text { Defectos óseos, articulares } 0 \\
\text { musculares }\end{array}$ & 4 & & 4,60 \\
\hline Polidactilia & & 4 & 100 \\
\hline Defectos multisistémicos & 3 & & 3,45 \\
\hline Síndrome de Wolf Hirschhorn & & 1 & 33,33 \\
\hline Síndrome de Goldenhar & & 1 & 33,33 \\
\hline Síndrome de Charge & & 1 & 33,33 \\
\hline Defectos cromosómicos & 2 & & 2,30 \\
\hline Síndrome de Down & & 2 & 100 \\
\hline Total & 87 & & \\
\hline
\end{tabular}

\section{Discusión}

Las anomalías congénitas son una importante causa de morbilidad y mortalidad, sobre todo durante el primer año de vida. El estudio de los factores relacionados con estas patologías, en especial aquellos que 


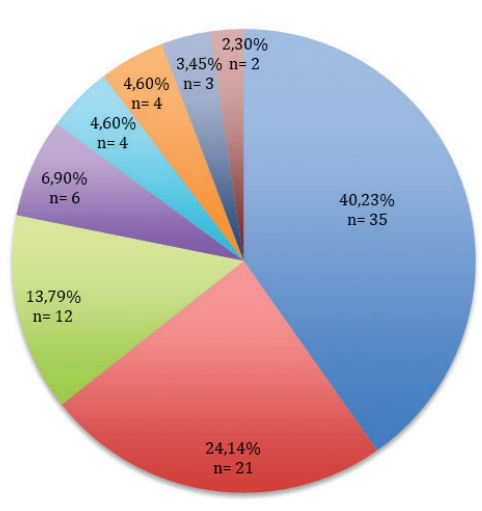

Defectos cardiovasculares

- Defectos Gastrointestinales $\rrbracket$ Defectos Urogenitales

ఐDefectos óseos, articulares o musculares $\equiv$ Defectos Multisistémicos
- Defectos craneales, faciales o cervicales

- Defectos Cromosómicos

Figura 1. Distribución de las frecuencias de anomalías congénitas por sistemas.

Tabla 3. Odds ratios obtenidas del análisis bivariado

\begin{tabular}{|c|c|c|}
\hline Posibles factores asociados & OR & IC95\% \\
\hline $\begin{array}{l}\text { Educación de la madre } \\
6 \text { años o más } \\
5 \text { años o menos }\end{array}$ & $\begin{array}{l}\text { Ref. } \\
2,40\end{array}$ & $1,23-4,68$ \\
\hline $\begin{array}{l}\text { Educación del padre } \\
6 \text { años o más } \\
5 \text { años o menos }\end{array}$ & $\begin{array}{l}\text { Ref. } \\
2,93\end{array}$ & $1,44-5,93$ \\
\hline $\begin{array}{l}\text { Consanguinidad } \\
\text { No } \\
\text { Sí }\end{array}$ & $\begin{array}{l}\text { Ref. } \\
6,37\end{array}$ & $0,75-54,07$ \\
\hline $\begin{array}{l}\text { Antecedente de cesárea en embarazos } \\
\text { previos } \\
\text { No } \\
\text { Sí }\end{array}$ & $\begin{array}{c}\text { Ref. } \\
3,3\end{array}$ & $1,76-6,42$ \\
\hline $\begin{array}{l}\text { Hemorragia en el primer trimestre } \\
\text { No } \\
\text { Sí }\end{array}$ & $\begin{array}{l}\text { Ref. } \\
6,15\end{array}$ & $1,32-28,63$ \\
\hline $\begin{array}{l}\text { Antecedente de malformaciones en } \\
\text { embarazos previos } \\
\text { No } \\
\text { Sí }\end{array}$ & $\begin{array}{l}\text { Ref. } \\
4,05\end{array}$ & $1,08-15,07$ \\
\hline $\begin{array}{l}\text { Endogamia } \\
\text { No } \\
\text { Sí }\end{array}$ & $\begin{array}{l}\text { Ref. } \\
0,95\end{array}$ & $0,51-1,76$ \\
\hline
\end{tabular}

IC95\%: intervalo de confianza del 95\%; OR: odds ratio. Análisis de regresión logística. Variable dependiente: malformaciones.

son modificables, es una de las claves para su prevención.

Durante el periodo en que se realizó la investigación se registraron 1477 nacimientos en el Hospital
Universitario San José de Popayán, entre los que se identificaron 87 casos de anomalías congénitas. Dentro de este grupo, las más frecuentes fueron defectos cardiovasculares $(40,23 \%)$, defectos del sistema urogenital $(24,14 \%)$ y defectos del sistema nervioso (13,79\%). Una información relevante es que estos datos difieren de los reportados por otros estudios en América Latina. Vega y Vizzuett ${ }^{10}$ refieren un $34,4 \%$ de malformaciones cardiovasculares en un hospital de tercer nivel en México, un 18,1\% de malformaciones craneales, faciales o cervicales, y un 13,8\% de malformaciones esqueléticas. En Uruguay ${ }^{3}$ se reportó un $52 \%$ de malformaciones del aparato genitourinario, un $28 \%$ de defectos cardiovasculares y un 16\% de malformaciones esqueléticas. En un estudio realizado en el Cauca por Bravo, et al. ${ }^{5}$, en 2012, se encontró que el 18,4\% de las anomalías congénitas correspondían a anomalías multisistémicas, el 14,3\% a anomalías gastrointestinales y el $11,2 \%$ a anomalías craneales, faciales 0 cervicales 5 .

Si se examina cada una de las anomalías congénitas reportadas, se encuentran en menor frecuencia los defectos de cierre del tubo neural, la espina bífida, la anencefalia y el encefalocele. Esta situación se presenta posiblemente por la fortificación de la harina con ácido fólico como parte de los programas de salud pública para la prevención primaria de dichas anomalías. En efecto, las tasas de defectos de cierre del tubo neural han disminuido de manera significativa $(p<0,02)$ en algunos países de Sudamérica ${ }^{11,12}$. 
En el análisis de las variables sociodemográficas se encontró que la baja escolaridad (5 años o menos) de ambos padres tuvo una relación significativa con la incidencia de anomalías congénitas. Datos similares han arrojado otros estudios en los que se encontró una asociación entre la baja escolaridad materna y el desarrollo de defectos del tubo neural (OR: 1,42; IC95\%: $1,19-1,70)^{13}$.

Estos resultados permiten inferir que las mujeres con alto riesgo de tener descendencia con defectos congénitos, como son aquellas con bajo nivel educativo, probablemente no tendrían acceso ni adherencia al control del embarazo ni a las ecografías obstétricas. Por tanto, tendrían menos probabilidades de tener un diagnóstico prenatal de anomalías congénitas, evitando así las intervenciones adecuadas para mejorar el pronóstico del embarazo y de los recién nacidos afectados ${ }^{14}$.

En el presente estudio no se observó asociación entre la consanguinidad y la presencia de anomalías congénitas, a diferencia de en otros, como el realizado en México con 1117 recién nacidos ${ }^{15}$, el cual reportó una asociación significativa para esta variable (OR: 2,4; IC95\%: 1,05-5,95), o el de Arabia Saudita con 2441 recién nacidos, que también reportó una asociación significativa entre la consanguinidad y las anomalías congénitas (OR: 1,5; IC95\%: 1,2-1,9) ${ }^{6}$. Este resultado probablemente se deba al pequeño tamaño muestral de nuestro estudio.

Un estudio llevado a cabo en $2018^{16}$ señaló una asociación significativa entre el antecedente de anomalías congénitas y la aparición de un segundo caso en la misma familia con una nueva anomalía congénita (OR: 59; IC95\%: 5,74-607,0), de modo similar a lo encontrado en la presente investigación. En otro estudio se ha reportado que la aparición de una anomalía congénita relacionada con defectos cromosómicos, con feto vivo o aborto, aumenta el riesgo de defectos cromosómicos en futuros embarazos ${ }^{17}$. Esto se ha reafirmado en otro estudio en Egipto ${ }^{18}$, en el que se observó una asociación significativa entre los antecedentes familiares de anomalías congénitas y su recurrencia.

En un estudio realizado en $2011^{19}$ se encontraron asociaciones significativas entre el tabaquismo materno y los defectos cardiovasculares o cardiacos (OR: 1,09; IC95\%: 1,02-1,17), los defectos musculoesqueléticos (OR: 1,16; IC95\%: 1,05-1,27), los defectos de reducción de extremidades (OR: 1,26; IC95\%: 1,15-1,39), los dedos faltantes o extra (OR: 1,18; IC95\%: 0,99-1,41), el pie zambo (OR 1,28; IC95\%: 1,10-1,47), la craneosinostosis (OR: 1,33; IC95\%: 1,03-1,73), los defectos faciales (OR: 1,19; IC95\%: 1,06-1,35), los defectos oculares
(OR: 1,25; IC95\%: 1,11-1,40), las hendiduras orofaciales (OR: 1,28; IC95\%: 1,20-1,36), los defectos gastrointestinales (OR: 1,27; IC95\%: 1,18-1,36), la gastrosquisis (OR: 1,50; IC95\%: 1,28-1,76), la atresia anal (OR: 1,20; IC95\%: 1,06-1,36), la hernia (OR: 1,40; IC95\%: 1,23-1,59) y los testículos no descendidos (OR: 1,13; IC95\%: 1,02-1,25).

Aunque se observó una relación entre la hemorragia del primer trimestre y las anomalías congénitas, los resultados no son concluyentes en cuanto a si el sangrado es parte del proceso patológico que condujo al resultado adverso o si es un síntoma de un proceso patológico completado y un concepto anormal resultante. En la literatura revisada no se encontraron otros estudios en los que se presente este mismo resultado.

Entre los factores estudiados, la cesárea previa se asoció con la presencia de anomalías congénitas. Este hallazgo también ha sido reportado por otros estudios, como el de Naghavi-Behzad, et al. ${ }^{20}$, en el que encontraron esta misma asociación (OR: 0,53; IC95\%: 0,37$0,75)$. Sin embargo, los mecanismos fisiopatológicos que puedan explicar esta relación aún no están claros $y$, por lo tanto, no se puede establecer una relación de causalidad. Algunos embarazos con anomalías congénitas terminan en cesáreas, y algunas condiciones maternas, como la obesidad, la preeclampsia y la diabetes gestacional, entre otras, se asocian a parto por cesárea y también pueden estar relacionadas con algunas anomalías congénitas, y de esta manera podría explicarse esta asociación.

Dentro de las fortalezas de la presente investigación cabe mencionar el instrumento utilizado, la herramienta del ECLAMC, estandarizada y validada para el diagnóstico y la caracterización de la población con anomalías congénitas ${ }^{7}$ Sumado a esto, también se cumplió con el tamaño muestral, y se contó con un experto en la parte diagnóstica del caso (médico genetista), complementándose con la realización de las ayudas diagnósticas posnatales requeridas para la confirmación del diagnóstico.

Con respecto a las debilidades, se tiene en consideración que las medidas de asociación corresponden a datos regionales (área de influencia del Hospital Universitario San José) y que al realizar modelos de análisis bivariado no se incluyeron otras variables que pudieran ser potenciales confusores. Podría también considerarse la posibilidad de un sesgo de información en cuanto a las variables de consanguinidad y endogamia, debido al bajo nivel educativo y cultural de la población estudiada, o a que la endogamia es una 
práctica censurada socialmente, por lo cual no se reconoce de manera abierta.

\section{Conclusiones}

Los resultados de los estudios llevados a cabo con el fin de dilucidar los diferentes factores de riesgo asociados con anomalías congénitas no son del todo consistentes y varían considerablemente entre diferentes regiones.

Los principales factores de riesgo asociados a anomalías congénitas encontrados en este estudio se deben a condiciones socioculturales, como el bajo nivel educativo tanto de la madre como del padre, y a condiciones biológicas y médicas de la madre, como la hemorragia del primer trimestre y el antecedente de anomalías congénitas. Aunque algunos de estos factores requieren ser estudiados a profundidad, se deben crear estrategias de intervención para los factores de riesgo prevenibles, como mejorar el acceso a la educación, identificar tempranamente las patologías de las mujeres en edad reproductiva y durante el embarazo, y realizar estudios y seguimiento a las mujeres con antecedente de anomalías congénitas.

\section{Financiación}

Los autores declaran que se usaron recursos propios para la financiación.

\section{Conflicto de intereses}

Los autores manifiestan no tener conflicto de intereses.

\section{Responsabilidades éticas}

Protección de personas y animales. Los autores declaran que para esta investigación no se han realizado experimentos en seres humanos ni en animales.

Confidencialidad de los datos. Los autores declaran que han seguido los protocolos de su centro de trabajo sobre la publicación de datos de pacientes.

Derecho a la privacidad y consentimiento informado. Los autores han obtenido el consentimiento informado de los pacientes y/o sujetos referidos en el artículo. Este documento obra en poder del autor de correspondencia.

\section{Bibliografía}

1. Zarante I, Franco L, López C, Fernández N. Frequencies of congenital malformations: assessment and prognosis of 52,744 births in three cities of Colombia. Biomedica. 2010;30:65-71.

2. Zarate AM, García G, Zarante I. Evaluación de factores de riesgo asociados con malformaciones congénitas en el programa de vigilancia epidemiológica de malformaciones congénitas (ECLAMC) en Bogotá entre 2001 y 2010. Univ Médica. 2016;53:11-25.

3. Bonino DA, Gómez P, Cetraro L, Etcheverry G, Pérez W. Malformaciones congénitas: incidencia y presentación clínica. Arch Pediatr Urug. 2006;77:225-8.

4. Aviña Fierro JA, Tastekin A. Malformaciones congénitas: clasificación y bases morfogénicas. Rev Mex Pediatr. 2008;75:71-4

5. Bravo-Gallego LY, Teherán-Bravo JM, Pantoja-Chamorro FI, Díaz-Castro R, Acosta-Aragón MA. Factores asociados a anomalías congénitas en neonatos del Cauca. Pediatria (Santiago). 2012;45:47-58.

6. Majeed-Saidan MA, Ammari AN, AlHashem AM, Al Rakaf MS, Shoukri MM, Garne E, et al. Effect of consanguinity on birth defects in Saudi women: Results from a nested case-control study. Birth Defects Res A Clin Mol Teratol. 2015;103:100-4.

7. ECLAMC. Manual operacional. 2009. Disponible en: http://www.eclamc.org/.

8. Sheridan E, Wright J, Small N, Corry PC, Oddie S, Whibley C, et al. Risk factors for congenital anomaly in a multiethnic birth cohort: An analysis of the Born in Bradford study. Lancet. 2013;382:1350-9.

9. Ramírez-Cheyne J, Pachajoa H, Ariza Y, Isaza C, Saldarriaga W. Defectos congénitos en un hospital de tercer nivel en Cali, Colombia. Rev Chil Obstet Ginecol. 2015;80:442-9.

10. Vega Valdés A, Vizzuett Martínez R. Frecuencia de malformaciones congénitas en un hospital general de tercer nivel. Rev Mex Pediatr. 2005;72:70-3.

11. Castilla EE, Orioli IM, López-Camelo JS, Dutra M da G, Nazer-Herrera J. Preliminary data on changes in neural tube defect prevalence rates after folic acid fortification in South America. Am J Med Genet. 2003;123A: 123-8.

12. Nazer J, Cifuentes L, Aguila A, Juárez ME, Cid MP, Godoy ML, et al. Efecto de la fortificación de la harina con ácido fólico sobre la evolución de las tasas de prevalencia al nacimiento de malformaciones congénitas en los hospitales chilenos del ECLAMC. Rev Med Chile. 2007;135:198-204.

13. Jia S, Wei X, Ma L, Wang Y, Gu H, Liu D, et al. Maternal, paternal, and neonatal risk factors for neural tube defects: a systematic review and meta-analysis. Int J Dev Neurosci. 2019;78:227-35.

14. Saldarriaga-Gil W, Ruiz-Murcia FA, Fandiño-Losada A, Cruz-Perea ME, Isaza de Lourido C. Evaluation of prenatal diagnosis of congenital anomalies diagnosable by prenatal ultrasound in patients in neonatal intensive care units of Cali, Colombia. Colomb Medica. 2014;45:32-8.

15. Pinto Escalante D, Castillo Zapata I, Ruiz Allec D, Ceballos Quintal JM. Espectro de malformaciones congénitas observadas en recién nacidos de progenitores consanguíneos. An Pediatr. 2006;64:5-10.

16. Ameen SK, Alalaf SK, Shabila NP. Pattern of congenital anomalies at birth and their correlations with maternal characteristics in the maternity teaching hospital, Erbil city, Iraq. BMC Pregnancy Childbirth. 2018;18:501.

17. El Koumi MA, AI Banna EA, Lebda I. Pattern of congenital anomalies in newborn: a hospital-based study. Pediatr Rep. 2013;5:e5

18. Shawky RM, Sadik DI. Congenital malformations prevalent among Egyptian children and associated risk factors. Egypt J Med Hum Genet. 2011;12:69-78.

19. Hackshaw A, Rodeck C, Boniface S. Maternal smoking in pregnancy and birth defects: a systematic review based on 173687 malformed cases and 11.7 million controls. Hum Reprod Update. 2011;17:589-604.

20. Naghavi-Behzad M, Alizadeh M, Azami S, Foroughifar S, Ghasempour-Dabbaghi K, Karzad N, et al. Risk factors of congenital heart diseases: a case-control study in Northwest Iran. J Cardiovasc Thorac Res. 2013;5:5-9. 